\title{
When do microsaccades follow spatial attention?
}

\author{
Jochen LAUbrock AND REINHOLD KLIEGL \\ University of Potsdam, Potsdam, Germany \\ Martin Rolfs \\ University of Potsdam, Potsdam, Germany \\ and CNRS and Université Paris Descartes, Paris, France \\ AND \\ RALF ENGBERT \\ University of Potsdam, Potsdam, Germany
}

\begin{abstract}
Following up on an exchange about the relation between microsaccades and spatial attention (Horowitz, Fencsik, Fine, Yurgenson, \& Wolfe, 2007; Horowitz, Fine, Fencsik, Yurgenson, \& Wolfe, 2007; Laubrock, Engbert, Rolfs, \& Kliegl, 2007), we examine the effects of selection criteria and response modality. We show that for Posner cuing with saccadic responses, microsaccades go with attention in at least $75 \%$ of cases (almost $90 \%$ if probability matching is assumed) when they are first (or only) microsaccades in the cue-target interval and when they occur between 200 and $400 \mathrm{msec}$ after the cue. The relation between spatial attention and the direction of microsaccades drops to chance level for unselected microsaccades collected during manual-response conditions. Analyses of data from four cross-modal cuing experiments demonstrate an above-chance, intermediate link for visual cues, but no systematic relation for auditory cues. Thus, the link between spatial attention and direction of microsaccades depends on the experimental condition and time of occurrence, but it can be very strong.
\end{abstract}

Eye movements and visual attention are intimately related. The major function of saccadic eye movements is to move objects of interest into the fovea, the retinal region of highest acuity, for close inspection during the following fixation (Findlay \& Gilchrist, 2003). Fixating an object means overtly attending to it. However, attention can also be covert - that is, dissociated from fixation position. When covert shifts of attention are induced with a centrally presented cue, responses to targets subsequently appearing at the cued peripheral location (valid-cue trials) are faster than responses to targets at the opposite location (invalid-cue trials; Posner, 1980; Posner, Snyder, \& Davidson, 1980). With the phrase "covert shifts of attention," reference is made to the absence of large saccadic eye movements during the cue-target interval (CTI). Whereas covert attention shifts are by definition not accompanied by overt saccades, there is evidence that saccades are obligatorily preceded by covert shifts of attention (i.e., processing at the saccade target is enhanced before saccade execution; Deubel \& Schneider, 1996; Hoffman \& Subramaniam, 1995; Kowler, Anderson, Dosher, \& Blaser, 1995).

A measure that can be used to track the deployment of covert attention may be useful in a number of contexts. Given the close relationship between saccades and visual attention, one might wonder whether traces of covert attention shifts can be detected in oculomotor activity during fixations. Since the absence of saccades during covert attention shifts does not imply the absence of fixational eye movements, microsaccades - small saccade-like movements with amplitudes $<1^{\circ}$ that occur during attempted ocular fixation (see Engbert, 2006, for a review) - have been proposed as a measure of covert attention. Given the result that microsaccades and saccades are probably regulated by the same physiological structures at the level of the superior colliculus (SC; Hafed, Goffart, \& Krauzlis, 2009; Rolfs, Kliegl, \& Engbert, 2008) and downstream (van Gisbergen, Robinson, \& Gielen, 1981), a relationship between covert attention and microsaccades may not be particularly surprising. In fact, evidence in favor of such a relationship has been presented: A considerable amount of research has demonstrated effects of attentional cue presentation on rate and direction of microsaccades (Corneil, Munoz, Chapman, Admans, \& Cushing, 2008; Engbert \& Kliegl, 2003; Galfano, Betta, \& Turatto, 2004; Gowen, Abadi, Poliakoff, Hansen, \& Miall, 2007; Hafed \& Clark, 2002; Kohama \& Usui, 2002; Laubrock, Engbert, \& Kliegl, 2008; Turatto, Valsecchi, Tamè, \& Betta, 2007).

A number of physiological control loops at several levels converge on the SC. For example, there is the low-level, reflexive, retino-tectal loop bypassing even the lateral geniculate nucleus. At higher levels, the SC receives cortical input from perceptual areas, from parietal cortex, and

J. Laubrock, laubrock@uni-potsdam.de 
from the frontal eye fields. Given this variety of inputs, it can be expected that the relationship (if any) between microsaccades and covert attention is not determined by a single process. Indeed, microsaccades may also result from other, not primarily attention-related processes (see Rolfs, 2009, for a comprehensive review), such as fixation control (e.g., Engbert \& Kliegl, 2004; Mergenthaler \& Engbert, 2007), perceptual disambiguation (e.g., Cui, Wilke, Logothetis, Leopold, \& Liang, 2009; Laubrock, Engbert, \& Kliegl, 2008; Starzynski \& Engbert, 2009; Troncoso, Macknik, Otero-Millan, \& Martinez-Conde, 2008; van Dam \& van Ee, 2006), and perceptual fading (e.g., Engbert \& Mergenthaler, 2006; Hsieh \& Tse, 2009; Martinez-Conde, Macknik, Troncoso, \& Dyar, 2006; Troncoso, Macknik, \& Martinez-Conde, 2008), suggesting a less than perfect agreement between the direction of attention and the direction of microsaccades.

Thus, we need to know more precisely about the inputoutput relations of the attention-microsaccade system in order to make microsaccades a useful measure of covert attention. To achieve this, it is necessary to collect data on the microsaccadic response in tasks known to affect covert attention. Empirically, whereas strong microsaccade rate modulations are observed even for irrelevant visual and auditory stimuli (Rolfs et al., 2008), effects on microsaccade direction depend on when in the CTI the microsaccade occurs, and are sensitive to a variety of experimental manipulations (overview in Rolfs, 2009), relating, for example, to the type of cue (arrow or color, Engbert \& Kliegl, 2003; central or peripheral, Gowen et al., 2007; Laubrock, Engbert, \& Kliegl, 2005), to the modality of the cue or the target (visual or auditory; Rolfs, Engbert, \& Kliegl, 2005), or to the type of response required (manual or saccadic; Laubrock, Engbert, Rolfs, \& Kliegl, 2007). There appears to be a strong link between the orienting system and microsaccade rate, and a weaker link between higher level attentional systems and microsaccade direction. Importantly, these effects are not purely cue driven. For example, an arrow cue biases microsaccade direction only under attention-shift instructions (to detect change in the periphery), but not under instructions to detect a central change (Engbert \& Kliegl, 2003).

Given these results, Horowitz, Fine, Fencsik, Yurgenson, and Wolfe's claim (2007, p. 356) that "fixational eye movements are not an index of covert attention" was a bit of a surprise to us. Whereas we think that this conclusion may be premature, clearly, the relationship between attention and microsaccades needs to be more fully specified. The present article aims at contributing to a closer specification of when and how microsaccades are affected by attention. Here we investigate the effect of response modality and of different selection criteria on the relationship between microsaccades and attention in the Posner cuing task. We also present a reanalysis of the effects of visual and auditory cues on attention shifts toward visual or auditory targets (Rolfs et al., 2005).

The background of the present research is a stimulating exchange about the question of whether fixational eye movements - in particular, microsaccades - can serve as an indicator of spatial attention shifts at all (Horowitz,
Fencsik, Fine, Yurgenson, \& Wolfe, 2007; Horowitz, Fine, et al., 2007; Laubrock et al., 2007). Horowitz, Fencsik, et al. based their conclusion that "the predictive power of microsaccades is, for practical purposes, negligible" (2007, p. 367) on two findings. First, they did not observe a large microsaccade-target congruency (MTC) effect on reaction times (RTs) in addition to the cue validity effect. In the present article, we argue that an index of attention must be related to attention, but need not produce effects in addition to the effects of attention. Imagine that we had lost part of the protocol of the experimental design and wanted to guess whether the cue on a given trial was valid or not. If this judgment is aided by knowledge of MTC, then microsaccades can be taken as indicators of attention. Thus, we predict faster RTs on trials with target-congruent microsaccades than on trials with target-incongruent microsaccades.

The strength of the relation between microsaccade parameters and RT varies strongly with experimental manipulations and microsaccade time of occurrence (Kliegl, Rolfs, Laubrock, \& Engbert, 2009). For example, microsaccades occurring shortly before the response drastically increase RT. Horowitz, Fine, et al. (2007) used a fairly wide time window $(400 \mathrm{msec})$, thereby potentially including late microsaccades, and also averaging over the effects of sequences of microsaccades with opposite directions. The robust microsaccadic rate modulations suggest that effects of attention will strongly depend on time relative to cue presentation. We here suggest that mainly microsaccades occurring in a relatively circumscribed interval about 200-400 msec after cue presentation are modulated by attention shifts in Posner-type cuing tasks (see also Engbert, 2006). We predict that if microsaccades in the CTI are taken from that interval, they will be related to RT. If, on the other hand, microsaccades are selected from a period later in the trial, then they may not be related to performance at all, or may even adversely affect it.

Second, Horowitz, Fine, et al. (2007) argued that sometimes attention might go the wrong way. They predicted that on these "attentional mistake" trials, if microsaccades indicated the direction of attention, performance should be better when microsaccades point toward the target than when they point away from the target. Empirically, however, they observed slower RTs in this case. In a response (Laubrock et al., 2007), we showed this result to be an artifact of trial selection. Figure 1 illustrates why. The root (left part) of the probability tree reflects the experimental design (neutral cues are left out for simplicity): The validity of arrow cues was $v=.80$, and .20 of trials were invalid. Moving to the right, we assume that attention follows these cues with probability $w$. Thus "attentional mistakes" occur with probability $1-w$. The products of the respective path probabilities yield estimates that attention is located either at the upcoming target (green ovals) or opposite to it (red ovals), given valid and invalid cues. ${ }^{1}$

Our main theoretical interest concerns the unknown probability $x$ with which microsaccades follow spatial attention. As we have argued above, it is likely that the relationship between microsaccade and attention is not perfect - that is, $x<1.00$ - and thus, with probability 


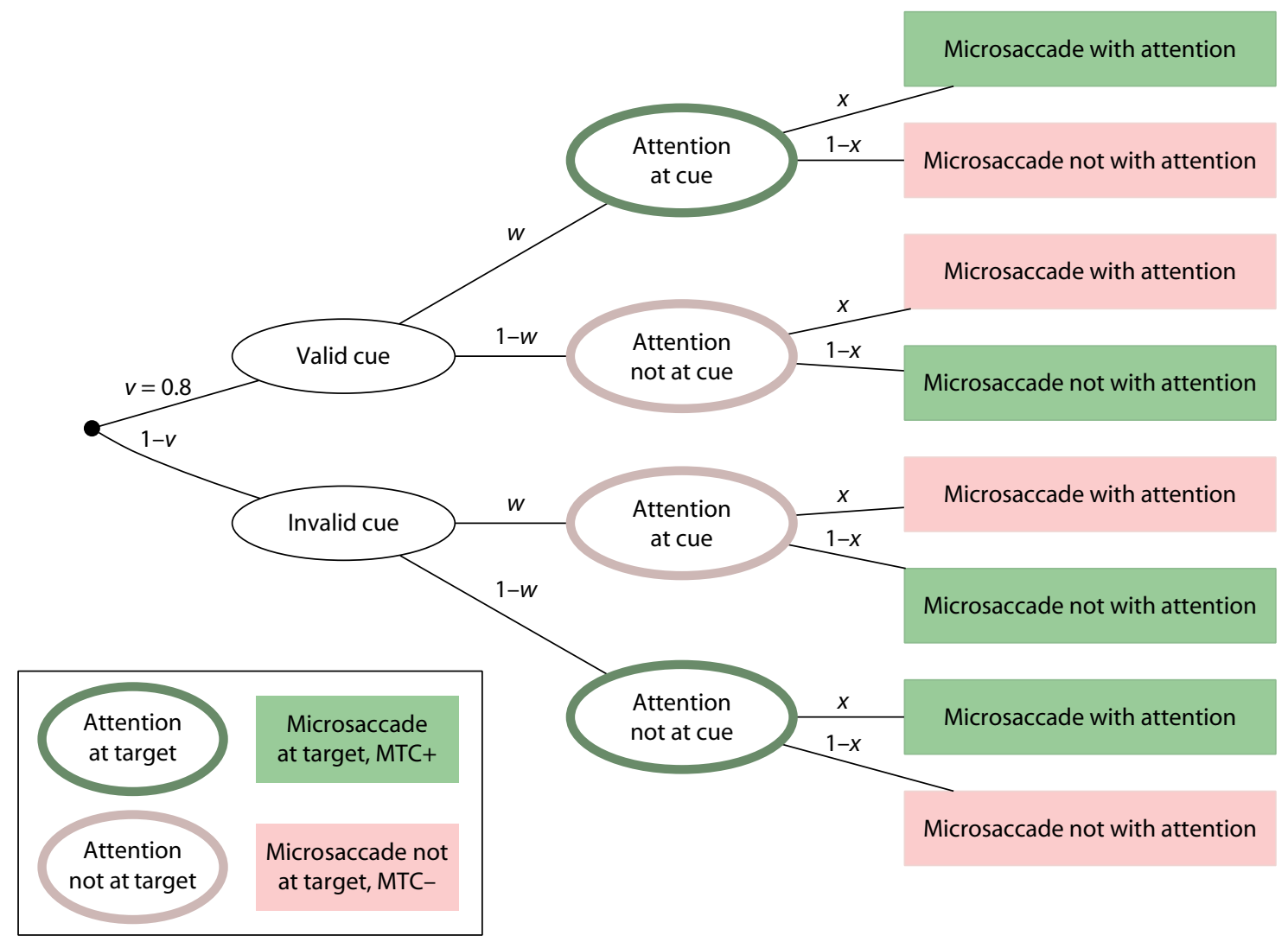

Figure 1. Multinomial tree model about the relation between the direction of spatially cued attention, cue validity, and direction of microsaccades. Neutral cues are omitted to avoid clutter. Validity for direction cues was fixed at $v=.80$. The parameter $w$ captures assumptions about attentional strategies - that is, about the probability that attention is indeed shifted in accordance with the cue; the range of reasonable values is between $w=v$ (probability matching) and $w=1.00$ (optimal attention shifts). The legend in the lower left corner explains the shape and color codes: Colors classify attention (ovals) or microsaccades (rectangles) with respect to the target; the dark gray and light gray colors indicate shifts toward and away from the target, respectively. At the behavioral level, we can only observe whether microsaccades are target congruent (dark gray rectangles) or target incongruent (light gray rectangles), because we cannot directly observe where attention is shifted. For example, on valid-cue trials (upper branch), we can only observe whether a microsaccade is directed at the target (dark gray rectangles) or not (light gray rectangles), but not whether attention is directed at the target (dark gray oval) or not (light gray oval). Hence, observed microsaccade-target congruency contains a mixture of instances involving attention at the target and away from the target. However, the observed probabilities constrain the possible values of the latent probabilities $x$ and $w$, because they are the sum of the products of the path probabilities leading to the rectangles of the given color. We are primarily interested in determining boundaries for $x$, the probability that the direction of attention determines the direction of microsaccades. These could indicate, for example, no relation $(x=$ $.50)$, an intermediate relation $(x=.75)$, or a deterministic link $(x=1.00)$. MTC, microsaccade-target congruency.

$1-x$ there are also cases in which microsaccades are not an indicator of attention. Adding these branches to the tree, we arrive at the set of possible terminal outcomes. However, these outcomes are still not directly observable. Instead, what is observable are microsaccades directed toward (MTC+, green boxes) or away from (MTC-, light red boxes) the target.

The selection of trials employed by Horowitz, Fine, et al. (2007) ignores the fact that for $x<1.00$, two paths lead to the empirically observable outcome "microsaccades not at target" (MTC-) in the upper branch of Figure 1. The associated probabilities are obtained by multiplication: $p_{1}=v w(1-x)$ and $p_{2}=v(1-w) x$. Note that only $p_{2}$ contains the "attentional mistake" cases Horowitz, Fine, et al. intended to analyze, whereas in $p_{1}$, attention is actually at the target, only the microsaccade is not. Indeed, by sym- metry, if $w>x$ (a mild boundary condition by no means implying that $x=.5$ ), then the selection on valid-cue trials will be dominated by cases in which attention is at the cued target, but microsaccades do not follow attention.

Importantly, "attentional mistake" cases as defined by Horowitz, Fine, et al. (2007) — that is, with microsaccades toward the target (MTC + ), but away from the cue - come exclusively from invalid-cue trials, whereas "attentional mistake" cases with microsaccades away from both the target (MTC-) and the cue come exclusively from validcue trials. Because the observable outcomes are determined by attention rather than by "attentional mistakes" in the paths $v w(1-x)$ and $(1-v) w(1-x)$, the contrast of RTs from these outcomes will also reflect this difference; hence, it is not particularly surprising that RT is faster for valid-cue, MTC-, than for invalid-cue, MTC + , cases. 
Horowitz, Fencsik, et al. (2007) accepted this rationale but cleverly noticed that, although $w$ and $v$ are not directly observable, we can observe the probability of microsaccades directed away from the target on valid-cue trials, $p=p_{1}+p_{2}=v(w+x-2 w x)$. We know that $v=.80$ (cue validity was set to $80 \%$ in the experiment), so any observed $p$ is compatible with a line through $w x$ space. Thus, we can compute a probability contour constraining values of delineating assumptions about $x$ and $w$ that are compatible with observed values of $p$. Indeed, the derivation of contours inspired the multinomial processing tree model reported in the article. Figure 2 illustrates how the observed probability of target-congruent microsaccades given valid cues (contours) constrains the possible values for the unknown probabilities that attention truly follows a spatial cue ( $y$-axis) and that microsaccades follow attention ( $x$-axis). Horowitz, Fencsik, et al. derived individual contours for their 3 subjects. Our reconstruction of the average of that contour is plotted in the left panel of Figure 2. Horowitz, Fencsik, et al. also inserted the prediction by Laubrock et al. (2007). Clearly, the mismatch between prediction and data could hardly be any larger: Apparently, their data indicate that microsaccade direction is completely independent of the direction of spatial attention.

We reanalyzed our 2007 data and found that they were not incompatible with the Laubrock et al. (2007) predic- tions. What are the conditions responsible for such large differences between two very similar experiments? We see three possible differences. First, not all microsaccades might be related to attention; therefore, a selection based on temporal interval might be needed to reveal attentional effects. Second, there were differences in procedure; in particular, Horowitz, Fine, et al. (2007) used a dual-Purkinje eyetracker, whereas Laubrock et al. (2007) used a video-based EyeLink II system. Third, there may be differences between subjects; this is potentially relevant because authors of both Laubrock et al. (2007) and Horowitz, Fine, et al. were also subjects in the studies, and we do not know to what extent microsaccades are affected by strategies. Although we cannot address the second point with this article, we collected new data with naive subjects to address the third issue, and varied selection criteria to address the first. Finally, the present study used a shorter CTI than did Laubrock et al. (2007) to reduce noise in the measurement of the cue validity effect in RTs, but an otherwise very similar experimental design.

\section{METHOD}

\section{Subjects}

Twenty students from the University of Potsdam received $€ 42$ or course credit for their participation in the experiment, comprising six sessions of about 45 min each. Two subjects were excluded from
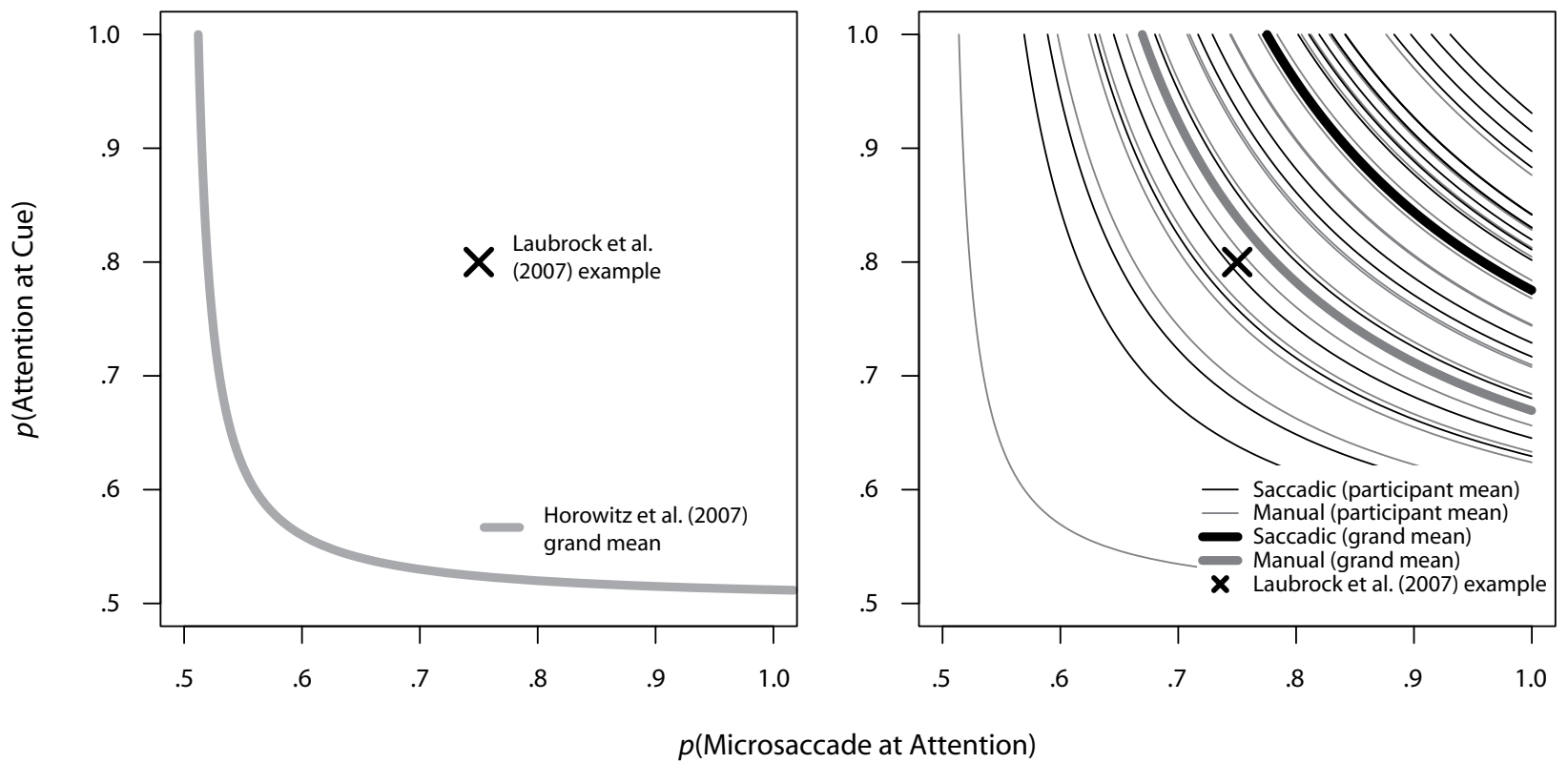

Figure 2. Each curve represents the observed probability of target-incongruent microsaccades given valid cues, and shows how this constrains the possible values for the unknown probabilities that attention followed the spatial cue ( $y$-axis) and that microsaccades followed attention ( $x$-axis). Curves are constructed following the analysis presented in Laubrock et al. (2007) and Horowitz, Fencsik, et al. (2007); see the text for details. (Left) Reconstruction of Horowitz, Fencsik, et al. (2007) and Horowitz, Fine, et al. (2007) results. (Right) New data. Each thin curve represents a single subject's probability, based on first microsaccades occurring 200-400 msec after cue presentation. Curve color distinguishes between saccadic (black) and manual (gray) responses. Thick lines represent mean probabilities across subjects. Assuming probability matching for attention following a cue $(p=.80)$ and an intermediate probability for microsaccades following the direction of attention $(x=.75)$, the manual-response curve would nearly pass through the point labeled "prediction by Laubrock et al. (2007)," which was included to facilitate comparison with Figure 1 from Horowitz, Fencsik, et al. (2007, p. 368; note that the point there erroneously exchanged coordinates). After visual comparison, only 1 of our subjects (represented by the curve in the lower left corner) produced a cue congruency effect on microsaccade direction that was as small as the average reported by Horowitz, Fencsik, et al. (2007). 
the analyses, 1 because of equipment failure, and the other because of a very high number of errors.

\section{Apparatus}

Each subject was seated in a silent and darkened room with his or her head positioned on a chinrest, $50 \mathrm{~cm}$ in front of a computer screen. Stimuli were presented on a 19-in. EYE-Q 650 CRT (resolution of $1,024 \times 768$ pixels or $36^{\circ} \times 27^{\circ}$ of visual angle; refresh rate $100 \mathrm{~Hz}$ ). Eye-position data were recorded and available online using a head-mounted EyeLink II system (SR Research, Osgoode, ON, Canada) with a sampling rate of $500 \mathrm{~Hz}$ and a noise-limited spatial resolution better than $0.01^{\circ}$. An Apple Power Macintosh G4 computer controlled stimulus display and response collection, using MATLAB (MathWorks, Natick, MA, USA) with the Psychophysics (Brainard, 1997; Pelli, 1997) and Eyelink (Cornelissen, Peters, \& Palmer, 2002) toolboxes. Manual responses were mapped to the left control key (left) or the right arrow key (right) on a standard Apple USB keyboard.

\section{Design}

In a factorial design, the experimental factors of response modality (manual, saccadic) and cue validity (valid, invalid, neutral with a proportion of $4: 1: 1$, respectively) and the control variable of target location (left, right) were varied within subjects. The experiment lasted for six sessions of 312 trials each (plus repeated trials; see below). Within each session, response modality was blocked, leading to 156 trials each with manual and saccadic responding. The order of blocks was switched between sessions and balanced across subjects. Cue validity and target location were randomized within blocks.

\section{Procedure}

A trial started with presentation of a central fixation cross $\left(.73^{\circ}\right.$ side length) and a fixation check, which required gaze to be within a square of $2^{\circ}$ side length at the screen center. Following successful fixation, the fixation cross remained on screen during a fixation period (1.5-2 sec; all randomized timings here were drawn from a uniform distribution). Next, the fixation cross turned into a cue (two lines were added to the cross, connecting its vertical wings to one of the horizontal wings, thereby creating an arrow to the left or to the right), which remained on screen for the duration of the CTI $(0.5-1.5 \mathrm{sec})$. The target was a disk of $0.73^{\circ}$ diameter with its center presented at $12.4^{\circ}$ horizontal eccentricity, whose appearance on one of the sides of the screen served as a go signal. Fixation was continuously checked before target onset, requiring gaze to stay within a central $3^{\circ}$ square. The target remained on screen for $1 \mathrm{sec}$ or until response, whichever came earlier. Whereas subjects were informed that they had to fixate the central cross before target onset, instruction stressed that the primary task was to report target location. For saccadic responding, gaze was required to be detected for at least $200 \mathrm{msec}$ in a $3^{\circ}$ square centered on either of the possible target locations. The next trial started after an intertrial interval of $0.5 \mathrm{sec}$.

Invalid trials due to failed fixation at the beginning of the trial or during the trial before the time of the go signal were canceled, as were trials in which no response was detected. Canceled trials were repeated at the end of a block in a random order. Drift correction was performed every 12 trials, and the eyetracker was recalibrated after every 24 trials or whenever the fixation check failed repeatedly.

\section{Data Analysis}

Saccade detection. In saccade blocks, response saccades were detected offline using a new version (Engbert \& Mergenthaler, 2006) of the algorithm by Engbert and Kliegl (2003). Velocities were computed from subsequent samples in the series of eye positions recorded after target presentation. Saccades were detected in 2-D velocity space using thresholds for peak velocity (6 SD) and minimum duration (6 msec, or three data samples). The first saccade that landed at one of the two potential target regions span- ning $\pm 1.5^{\circ}$ around the target was defined as the response saccade. Saccade latency was defined as the latency between target presentation and response saccade onset. Manual responses were scored as incorrect if the wrong target location was reported; saccadic responses were scored as incorrect if they landed in the wrong target region.

Microsaccade detection. We used the same algorithm to detect microsaccades with amplitudes $<1^{\circ}$ in the interval from $50 \mathrm{msec}$ before cue onset to the (manual or saccadic) response. We considered only binocular microsaccades - that is, microsaccades detected in both eyes with temporal overlap of at least one data sample. Trials including saccades larger than $1^{\circ}$ prior to the response were discarded, as were trials with no responses and saccade latencies shorter than $70 \mathrm{msec}$. The 18 participants contributed a total of 34,082 trials, in which 37,181 microsaccades were detected.

Classification of type of microsaccade. For analyses of the relation between microsaccade occurrence and response times, microsaccades during the CTI were classified into three groups: (1) single microsaccades $(N=10,518)$ from trials in which only one microsaccade occurred during the CTI, (2) the first of several microsaccades $(N=8,220)$, and (3) the last of several microsaccades $(N=8,220)$ from trials in which more than one microsaccade occurred during the CTI. Note that groups 2 and 3 are based on the same trials. Therefore, differential effects of the direction of first and last microsaccades on RT cannot be due to differences in trial selection. On average, the horizontal directions of first and last microsaccades were negatively correlated $(r=-.389)$ (range between subjects: -.16 to -.67 ), and the average $N$ of trials per subject with more than one microsaccade was 443 . The mean times of occurrence were $363 \mathrm{msec}(S E=13 \mathrm{msec}), 273 \mathrm{msec}(S E=12 \mathrm{msec})$, and $561 \mathrm{msec}(S E=21 \mathrm{msec})$ after the cue for single, first, and last microsaccades, respectively.

\section{RESULTS}

\section{Reaction Times and Errors}

For the analysis of cue validity effects, mean RTs from correct trials were submitted to an ANOVA with cue validity (valid, neutral, invalid) and response modality (manual, saccadic) as within-subjects factors. Saccadic RTs were faster than manual RTs $[M=181$ vs. $335 \mathrm{msec}$; $\left.F(1,17)=227, M S_{\mathrm{e}}=2,823, p<.001\right]$. Cue validity had the expected effect, with valid cues leading to faster RTs $(236 \mathrm{msec})$ than neutral cues $(263 \mathrm{msec})$ or invalid cues $(279 \mathrm{msec})\left[F(2,34)=65, M S_{\mathrm{e}}=267, p<.001\right]$. Cue validity and response modality interacted, with manual RTs showing a greater cue validity effect (see Table 1 for means). Separate analyses for each response modality showed that cue validity had a significant effect within each response modality [manual, $F(2,34)=852, M S_{\mathrm{e}}=$ 254, $p<.001$; saccadic, $F(2,34)=16, M S_{\mathrm{e}}=106, p<$

Table 1

Means and Standard Errors (Between Subjects) for Reaction

Times (RTs; Keypresses or Saccade Onset Latencies) and Errors, Broken Down by Response Modality and Cue Validity

\begin{tabular}{llccccc}
\hline \multirow{2}{*}{$\begin{array}{l}\text { Response } \\
\text { Modality }\end{array}$} & Cue & \multicolumn{2}{c}{ RT $(\mathrm{msec})$} & & \multicolumn{2}{c}{$\%$ Error } \\
\cline { 3 - 4 } \cline { 6 - 7 } Malidity & $M$ & $S E$ & & $M$ & $S E$ \\
\hline \multirow{2}{*}{ Sanual } & Invalid & 369 & 12.8 & & 4.94 & 1.43 \\
& Neutral & 339 & 15.1 & & 2.30 & 1.24 \\
& Valid & 300 & 11.5 & & 1.45 & 1.18 \\
& Invalid & 188 & 5.5 & & 0 & - \\
& Neutral & 185 & 5.2 & & 0 & - \\
& Valid & 170 & 3.4 & & 0 & - \\
\hline
\end{tabular}


.001]. Planned comparisons with the neutral condition showed that valid cues produced a benefit for both manual and saccadic responses (all $p \mathrm{~s}<.001)$, whereas invalid cues had a cost only for manual $(p<.001)$ but not for saccadic responses $(F<1)$.

Error rates were generally small, and no errors at all were observed in the saccadic task, presumably due to the attention-capturing target combined with optimal stimulus-response mapping. Therefore, the analysis of cue validity on errors was restricted to manual responses, using a repeated measures ANOVA of mean error rate with the factor cue validity, which influenced error rate $\left[F(2,34)=9.36, M S_{\mathrm{e}}=.001, p=.005\right]$. Invalid cues caused more errors than neutral cues $[F(1,17)=8.09$, $\left.M S_{\mathrm{e}}=.002, p=.011\right]$, which in turn caused more errors than valid cues $\left[F(1,17)=6.93, M S_{\mathrm{e}}=2.1 \mathrm{E}-4, p=\right.$ .017]. In all subsequent analyses, only trials with correct responses were included.

\section{Microsaccade Amplitudes}

Microsaccades had characteristics similar to those reported in most of the recent literature (see MartinezConde, Macknik, Troncoso, \& Hubel, 2009, for an overview); that is, their amplitude was small (mean amplitude in the present study: 15.1 arcmin, first to third quartiles at 7.6, 13.1, and 15.1 arcmin) and they fell on the "main sequence" describing a linear relation of amplitude and peak velocity. However, the characteristics of microsaccades changed during the course of a trial. In particular, microsaccade amplitude varied with selection criteria: If a trial contained more than one microsaccade, then the amplitude of the first of several was a bit larger and the amplitude of the last of several was quite a bit smaller than the amplitude of microsaccades from trials with only a single microsaccade during the CTI. Figure 3 shows mi-

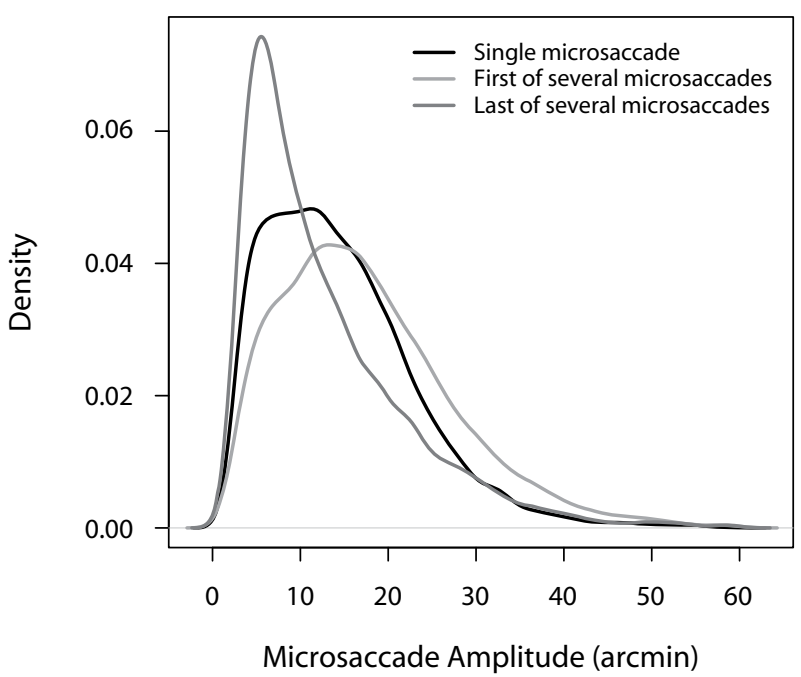

Figure 3. Distributions of microsaccade amplitudes broken down by type of microsaccade (single, first of several, last of several). Densities are collapsed across response modality because their shape was very similar. crosaccade amplitude distributions according to the different selection criteria.

\section{Microsaccade Rate and Direction}

The signature of microsaccade behavior is the development of their rate and orientation across the CTI. Figure 4 displays these statistics for target-congruent microsaccades (black lines) and target-incongruent microsaccades (gray lines) broken down by selection criterion (single, first of several, last of several) and cue validity (invalid, neutral, valid). As in all previous research (e.g., Engbert $\&$ Kliegl, 2003), the rates reveal an early suppression followed by a pronounced overshoot before a return to low rates. Especially around the peak, microsaccades in the cue direction are much more frequent than those against it. There are three new results in this figure. First, bimodality is restricted to single or first microsaccades, and is more pronounced for first than for single microsaccades; last microsaccades show a very different time course. This result will be important for the following analyses. Second, the timing of cue-congruent and cue-incongruent microsaccades differs for first or single microsaccades. Congruent microsaccades tend to occur shortly after the cue, whereas cue-incongruent microsaccades dominate later in the trial. Third, the likelihood of observing more than a single microsaccade in the CTI increases if a cueincongruent microsaccade occurs around the time of cue presentation (within $150 \mathrm{msec}$ ). The first two results were seen both for manual and saccadic response conditions, whereas the third was limited to saccadic responding.

\section{Empirical Microsaccade-Target Congruencies}

Focusing on a previously established temporal interval (see Engbert, 2006, for an overview) around the likely time of occurrence of attention-shift-related microsaccades (200-400 msec after cue), we computed the observed probabilities of target-congruent microsaccades on valid-cue trials. For the aggregated data of our subjects we obtained the result in the right panel of Figure 2. The observed probabilities clearly indicate that possible values of the probability that microsaccades follow attention are greater than .5. Depending on what one is willing to assume about the unknown probability $w$ that attention goes with the cue, the estimated probability that microsaccade direction goes with attention is between around .7 and 1.0. We conclude that under some conditions microsaccades obviously follow attention with above-chance probability. The probability is higher with saccadic than with manual responding, but it is above chance in both response conditions.

\section{Do Microsaccades Go With Spatial Attention?}

How do the empirical probabilities of target-congruent microsaccades under the various cue conditions compare with predictions of the multinomial tree model of Figure 1? The bold lines in Table 2 contain the predictions for target-congruent and target-incongruent microsaccade probabilities for valid, invalid, and neutral cues, given various assumptions about attentional strategy 


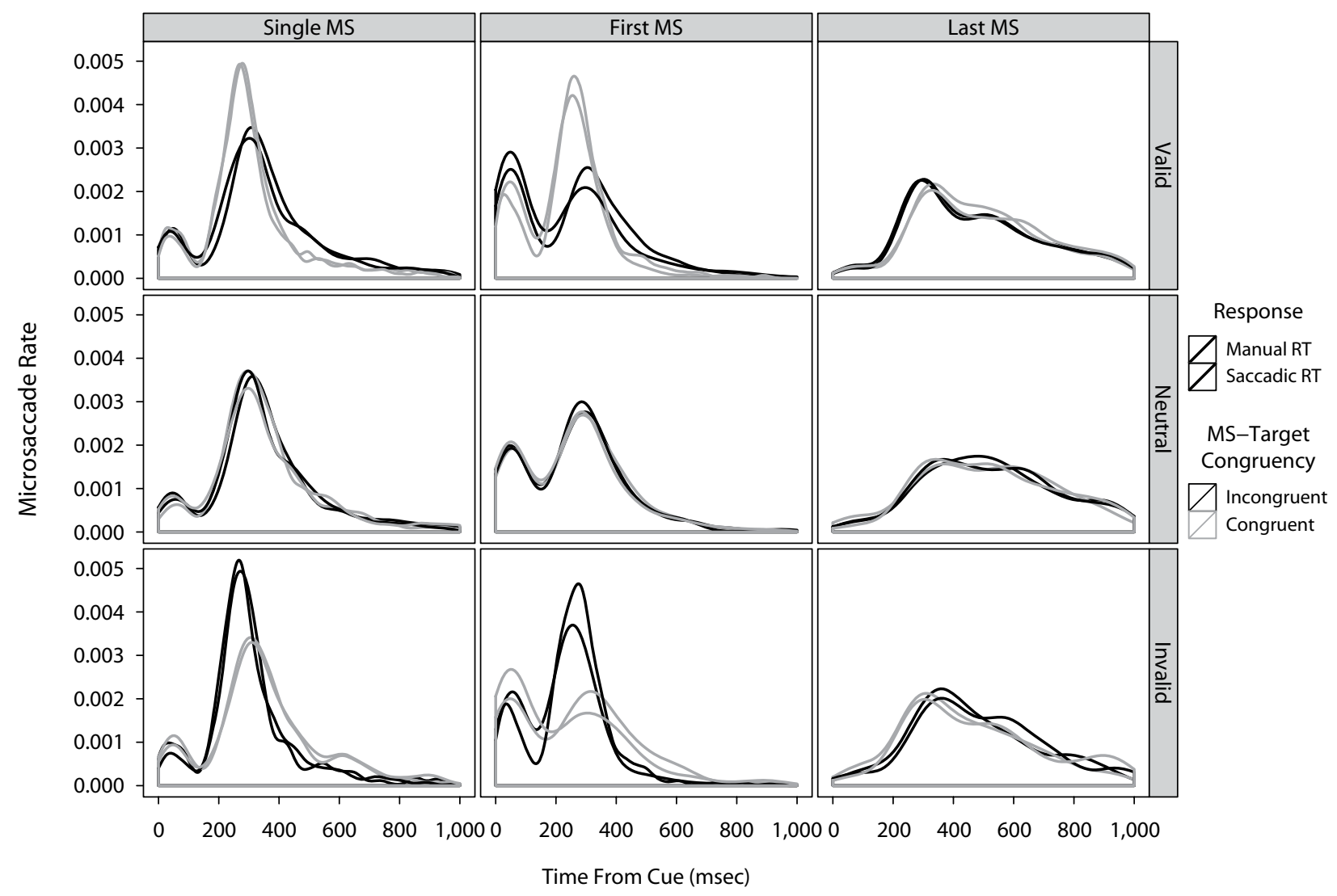

Figure 4. Microsaccade rates across the cue-target interval for target-congruent (gray lines) and target-incongruent (black lines) microsaccades broken down by type of microsaccades (panel columns: single, first of several, last of several), cue validity (panel rows: invalid, neutral, valid), and response modality (manual: thin lines; saccadic: thick lines). Note that target-incongruent rates for invalid cues (bottom row of panels) reflect cue-congruent rates. Thus, cues induce a pronounced peak in microsaccade rate at around $200 \mathrm{msec}$ in the cue-target interval. Graph produced with ggplot ( $R$ Development Core Team, 2009; Wickham, 2009). MS, microsaccade.

Table 2

Predicted (Bold Font) and Observed (Regular Font) Probabilities of TargetCongruent (MTC: +) and Target-Incongruent (MTC: - ) Microsaccades Following Valid, Invalid, and Neutral Cues for Multinomial Tree Model (See Figure 1)

\begin{tabular}{lcccccccr}
\hline & \multicolumn{3}{c}{ Valid } & \multicolumn{3}{c}{ Invalid } & \multicolumn{2}{c}{ Neutral } \\
& Cues & \multicolumn{2}{c}{ Cues } & Cues & $N$ \\
\hline MTC & + & - & + & - & + & - & \\
Model: $\boldsymbol{x}=\mathbf{1 . 0}, \boldsymbol{w}=\mathbf{1 . 0}$ & $\mathbf{. 6 7}$ & $\mathbf{. 0 0}$ & $\mathbf{. 0 0}$ & $\mathbf{. 1 7}$ & $\mathbf{. 0 8}$ & $\mathbf{. 0 8}$ & \\
Model: $\boldsymbol{x}=\mathbf{1 . 0}, \boldsymbol{w}=\mathbf{. 8}$ & $\mathbf{. 5 3}$ & $\mathbf{. 1 3}$ & $\mathbf{. 0 3}$ & $\mathbf{. 1 3}$ & $\mathbf{. 0 8}$ & $\mathbf{. 0 8}$ & \\
Saccadic $(200-400 ;$ single \& first MS) & .52 & .17 & .04 & .12 & .07 & .07 & 5,784 \\
Model: $\boldsymbol{x}=\mathbf{. 7 5}, \boldsymbol{w}=\mathbf{1 . 0}$ & $\mathbf{. 5 0}$ & $\mathbf{. 1 7}$ & $\mathbf{. 0 4}$ & $\mathbf{. 1 3}$ & $\mathbf{. 0 8}$ & $\mathbf{. 0 8}$ & \\
Saccadic (CTI; single \& first MS) & .46 & .22 & .06 & .10 & .08 & .08 & 10,504 \\
Model: $\boldsymbol{x}=\mathbf{. 7 5}, \boldsymbol{w}=\mathbf{. 8}$ & $\mathbf{. 4 3}$ & $\mathbf{. 2 3}$ & $\mathbf{. 0 6}$ & $\mathbf{. 1 1}$ & $\mathbf{. 0 8}$ & $\mathbf{. 0 8}$ & \\
Saccadic (CTI; single, first, last MS) & .40 & .27 & .07 & .09 & .08 & .08 & 15,293 \\
Manual (200-400; single \& first MS) & .40 & .27 & .07 & .10 & .08 & .08 & 5,042 \\
Manual (CTI; single \& first MS) & .36 & .32 & .07 & .09 & .08 & .09 & 9,972 \\
Manual (CTI; single, first, last MS) & .34 & .34 & .08 & .08 & .08 & .08 & 14,556 \\
Model: $\boldsymbol{x}=\mathbf{. 5}$ & $\mathbf{. 3 3}$ & $\mathbf{. 3 3}$ & $\mathbf{. 0 8}$ & $\mathbf{. 0 8}$ & $\mathbf{. 0 8}$ & $\mathbf{. 0 8}$ & \\
\hline
\end{tabular}

Note-Predicted probabilities for models are printed in bold, assuming the optimal observer model $(w=1.00)$ and the probability matching model $(w=.80)$, and three different values for the probability that microsaccades follow attention (no relation, $x=.50$; intermediate link, $x=.75$; deterministic link, $x=1.00$ ). Observed probabilities for saccadic and manual responses are listed in proximity to model predictions of best correspondence. Rows are sorted by the probability of observing target-congruent microsaccades on valid-cue trials. MTC, microsaccade-target congruency; CTI, cue-target interval; MS, microsaccade. 
$(w=.8, w=1.0)$ and microsaccade validity $(x=.5$, $x=.75, x=1.0$ ). The empirical probabilities are listed for different selection criteria. Rows are sorted by the probability of observing target-congruent microsaccades on valid-cue trials.

Manual-response blocks. Starting at the bottom of Table 2, we note a very close correspondence between the independence model $(x=.5)$ and all (unselected) microsaccades from manual-response blocks. This result replicates Horowitz, Fine, et al. (2007). The next two lines (1) leave out last microsaccades and, in addition, (2) select microsaccades only from a CTI time window of 200 $400 \mathrm{msec}$, placed around the peak rate visible in Figure 3. Clearly, for these selections the empirical probabilities are increasingly similar to the multinomial model assuming probability matching and an intermediate link between spatial attention and subsequent direction of microsaccade $(x=.75)$.

Saccadic-response block. The unselected sample of microsaccades from saccadic-response trials is quite similar to this intermediate-link model. If we include only single and first microsaccades from the 200- to 400-msec time window and again assume probability matching, then the empirical probabilities agree almost perfectly with the theoretical probabilities computed on the assumptions of a deterministic link between spatial attention and microsaccade direction.

Probability estimates that microsaccades go with attention. For a quantitative estimate of the probability that microsaccades indicate attention, we fit the multinomial model to each subject's data, simultaneously fitting the observed probabilities of MTC for all levels of cue validity. Technically, this was achieved by minimizing a cost function defined by the sum of the squared deviations of the empirically observed probabilities from the theoretically predicted probabilities of microsaccades directed at or away from the target (i.e., the terminal nodes in the model in Figure 1), assuming (1) Bayesian optimality; that is, observers always shift their attention with the cue, or (2) probability matching; that is, observers shift their attention with the cue proportional to cue validity. The parameter to be minimized was $x$, the probability that microsaccades follow attention. The model was fit to individual observers' data, and mean results are reported in Table 3.

We used this analysis to test the influences of response modality and of the selection criteria employed (i.e., which microsaccades to include in the analysis). Results indicate that both factors have an influence. First, microsaccades are more indicative of attention shifts for saccadic than for manual responses. Thus, the responsiveness of the oculomotor system to attentional cues is modified by response demands. Second, if the analysis is restricted to first or single microsaccades occurring $200-400 \mathrm{msec}$ after the cue, then even under the conservative assumption that subjects always shift their attention with the cue according to Bayesian optimality, the probability that microsaccades follow attention is relatively high $(x=.77$ for saccadic responding, $x=.61$ for manual responding). If subjects were choosing a probability-matching strategy,
Table 3

Probability That Microsaccades Went With Attention

\begin{tabular}{|c|c|c|c|c|}
\hline \multirow{2}{*}{$\begin{array}{c}\text { Response Condition and } \\
\text { Microsaccade Selection Criterion }\end{array}$} & \multicolumn{2}{|c|}{$\begin{array}{c}\text { Probability } \\
\text { Matching, } \\
w=.80 \\
\end{array}$} & \multicolumn{2}{|c|}{$\begin{array}{c}\text { Optimal } \\
\text { Attention } \\
\text { Shifting, } \\
w=1.00\end{array}$} \\
\hline & $M$ & $S E$ & $M$ & $S E$ \\
\hline Saccadic (200-400; single or first MS) & .88 & .08 & .77 & .10 \\
\hline Saccadic (200-400; first MS) & .86 & .08 & .77 & .10 \\
\hline Saccadic $(0-500$, single MS $)$ & .86 & .08 & .74 & .10 \\
\hline Saccadic (CTI; single MS) & .82 & .09 & .71 & .11 \\
\hline Saccadic (CTI; single or first MS) & .81 & .09 & .70 & .11 \\
\hline Saccadic (CTI; first MS) & .78 & .10 & .68 & .11 \\
\hline Saccadic (CTI; any MS) & .70 & .11 & .62 & .11 \\
\hline Saccadic $(500-1,000 ;$ single MS) & .57 & .12 & .54 & .12 \\
\hline Saccadic RT (CTI; last MS) & .38 & .11 & .43 & .12 \\
\hline Manual (200-400; first MS) & .72 & .11 & .65 & .11 \\
\hline Manual (200-400; single or first MS) & .66 & .11 & .61 & .12 \\
\hline Manual $(0-500 ;$ single MS $)$ & .61 & .11 & .57 & .12 \\
\hline Manual (CTI; first MS) & .59 & .12 & .55 & .12 \\
\hline Manual (CTI; single or first MS) & .58 & .12 & .55 & .12 \\
\hline Manual RT \& single MS & .58 & .12 & .55 & .12 \\
\hline Manual (CTI; any MS) & .54 & .12 & .53 & .12 \\
\hline Manual (CTI; last MS) & .46 & .12 & .47 & .12 \\
\hline Manual (500-1,000; single MS) & .38 & .11 & .43 & .12 \\
\hline
\end{tabular}

Note-Predicted probabilities $x$ that microsaccades went with attention, given the empirically observed microsaccade-target congruencies (MTCs), and assumptions about the probability $w$ that attention is directed by the cue (probability matching, $w=.80$; optimal attention shifts, $w=1.00$ ), for various microsaccade selection criteria. Predictions were derived per subject by minimizing the sum of squared deviations of observed MTC probabilities from predictions generated by the model (see Figure 1). Table cells contain the mean predicted probability across subjects and the associated standard error of proportions $(S E)$. Table rows are sorted by $x$ in decreasing order within each response modality. MS, microsaccade; CTI, cue-target interval.

the probabilities would be even higher $(x=.88$, saccadic; $x=.66$, manual).

If, on the other hand, all microsaccades within the CTI are included, then the link between microsaccades and attention appears to be rather weak (optimal: $x=$ .62 saccadic; $x=.53$ manual; probability matching: $x=$ .70 saccadic; $x=.54$ manual). This is at least partly due to inclusion of the last of several microsaccades, the direction of which is negatively correlated with the direction of the first of several microsaccades (see above), and therefore often directed away from the cue. Indeed, if only the last of several microsaccades or a single microsaccade late in the trial were inspected, then the conclusion would be that microsaccades are negatively correlated with attention $(x=$ .43 , for shift and saccadic or manual responses; $x=.38$, for probability matching and saccadic or manual responses).

\section{Microsaccade-Target Congruency As a Predictor of Reaction Times}

Can MTC recover some of the RT benefits introduced by the cues? If microsaccades are related to attention, then RTs on trials with target-congruent microsaccades should be shorter than on trials with target-incongruent microsaccades. We specified a linear mixed model (LMM) using restricted marginal likelihood estimates (R package lme4; Bates \& Maechler, 2009) to predict RT as a function of the following predictors (including interactions): MTC 
(congruent, incongruent), response modality (manual, saccadic), and session (linear and quadratic trends). Subjects were specified as a random factor. We included only first microsaccades occurring $200-400 \mathrm{msec}$ after cue presentation. In addition to response modality $(M=$ $-140, S E=3, t=-46)$ and the linear contrast of ses$\operatorname{sion}(M=-2,619, S E=372, t=-7)$, MTC $(M=-20$, $S E=3, t=-7)$ had a strong effect, indicating $20 \mathrm{msec}$ shorter RTs with target-congruent microsaccades. This was modulated by an interaction of MTC and response modality $(M=9, S E=3.9, t=2.3)$, meaning that the RT benefit of target-congruent saccades $(11 \mathrm{msec})$ was smaller for saccadic than for manual responding-which is likely due to a scaling of RT benefits with absolute RT. In summary, target congruency of first postcue microsaccades went along with an RT benefit.

\section{Visual-Auditory Cross-Modal Validation of Spatial Attention Effect}

As an additional check on the conditions under which the various predictions of the multinomial tree models hold, we reanalyzed data from four experiments reported by Rolfs et al. (2005; also Kliegl et al., 2009). In this study, visual or auditory peripheral cues preceded visual or auditory targets with a CTI of $1 \mathrm{sec}$. The task was a very difficult discrimination task using manual responses to red and green visual targets and to low- and high-pitched tones, respectively. Microsaccades were classified by whether their direction was congruent with the target location or not (using polar quadrants). Table 4 lists estimated probabilities that microsaccades went with attention, assuming either probability matching or optimal attention shifting, and for microsaccades selected according to different criteria. The first postcue microsaccades indicated attention shifts only if the cue was visual, but not if auditory cues were used (for which, if anything, there seems to be a negative correlation between the directions of early microsaccades and attention).

Figure 5 displays the $x$ values computed from the MTC probabilities of valid cues, separately for 200-msec bins of the CTI for each of the four experiments. For the visualcue conditions (panels VV and VA) there are distinct peaks for the MTC probability for the interval from 200 to $400 \mathrm{msec}$ with an $x$ value of about .75, and also effects in the subsequent interval indicating that the attention effect in this difficult task is more sustained than in the easy Posner-type cuing tasks reported above. In contrast, for auditory-cue conditions, the $x$ values hover around the chance level of .50 across the entire CTI.

\section{DISCUSSION}

The results establish a relatively tight relationship of early, cue-related microsaccades with the direction of spatial attention. There are experimental conditions and time windows during which microsaccades go in the direction of spatial attention more than $77 \%$ of the time (in the following, we will call this the "SA-MD link"), even under the conservative assumption that subjects always shift their attention with the cue. With the more liberal as-
Table 4

Predicted Probabilities $x$ That Microsaccades Went With Attention for the Cross-Modal Cuing Experiment (Rolfs et al., 2005)

\begin{tabular}{|c|c|c|c|c|}
\hline \multirow{2}{*}{$\begin{array}{c}\text { Cue Type and Microsaccade } \\
\text { Selection Criterion }\end{array}$} & \multicolumn{2}{|c|}{$\begin{array}{c}\text { Probability } \\
\text { Matching, } \\
w=.80 \\
\end{array}$} & \multicolumn{2}{|c|}{$\begin{array}{c}\text { Optimal } \\
\text { Attention } \\
\text { Shifting, } \\
w=1.00\end{array}$} \\
\hline & $M$ & $S E$ & $M$ & $S E$ \\
\hline Visual (200-400; single or first MS) & .74 & .10 & .65 & .11 \\
\hline Visual (200-400; first MS) & .69 & .11 & .65 & .11 \\
\hline Visual (CTI; first MS) & .62 & .11 & .57 & .12 \\
\hline Visual (CTI; single or first MS) & .61 & .11 & .57 & .12 \\
\hline Visual (0-500; single MS) & .57 & .12 & .54 & .12 \\
\hline Visual (CTI; single MS) & .57 & .12 & .54 & .12 \\
\hline Visual (CTI; any MS) & .56 & .12 & .53 & .12 \\
\hline Visual (500-1,000; single MS) & .47 & .12 & .48 & .12 \\
\hline Visual (CTI; last MS) & .44 & .12 & .46 & .12 \\
\hline Auditory (CTI; last MS) & .51 & .12 & .51 & .12 \\
\hline Auditory (CTI; single MS) & .51 & .12 & .50 & .12 \\
\hline Auditory (CTI; any MS) & .50 & .12 & .50 & .12 \\
\hline Auditory (CTI; first MS) & .49 & .12 & .49 & .12 \\
\hline Auditory (CTI; single or first MS) & .48 & .12 & .49 & .12 \\
\hline Auditory (200-400; single or first MS) & .47 & .12 & .48 & .12 \\
\hline Auditory (200-400; first MS) & .47 & .12 & .48 & .12 \\
\hline Auditory (500-1,000; single MS) & .46 & .12 & .48 & .12 \\
\hline Auditory $(0-500 ;$ single MS) & .46 & .12 & .48 & .12 \\
\hline
\end{tabular}

Note-The presentation format is like in Table 3. Assumptions about the probability $w$ that attention is directed by the cue (probability matching, $w=.80$; optimal attention shifts, $w=1.00$ ) were varied. Predictions were derived per subject by minimizing the sum of squared deviations of observed MTC probabilities from predictions generated by the model (see Figure 1). Table cells contain the mean predicted probability across subjects and the associated standard error of proportions $(S E)$. Table rows are sorted by $x$ in decreasing order within each cue type. MS, microsaccade; CTI, cue-target interval; MTC, microsaccadetarget congruency.

sumption that subjects shift spatial attention with a probability matching cue validity, the strength of the SA-MD link increases to almost $90 \%$.

Thus, counter to Horowitz, Fine, et al.'s (2007) claim, microsaccades can be quite reliable indicators of spatial attention. Note, however, that when we analyze the data in more aggregated form - using a wider time window, similar to the way Horowitz, Fine, et al. did — we replicate their result of a near absence of the SA-MD link. Obviously, we are only at the beginning of detailing the conditions under which the link is reliable, but our analyses do give information about some of the major constraints. We will discuss five of them.

First (single) microsaccade. The SA-MD link is probably strongest for the first (or only) microsaccade after the cue. This result was foreshadowed by a striking difference for the rate-direction signature of last microsaccades: They lack the suppression and rebound effect (see Figure 4). In contrast, there are microsaccade-related modulations of RTs that appear to be strongest for the last microsaccade in the CTI (Kliegl et al., 2009). Thus, temporal proximity is critical. Quite possibly, effects related to first and last microsaccades reflect different underlying mechanisms.

Last microsaccade. Although the effects are not very strong, effects observed in last microsaccades suggest that oculomotor activity later in the trial fluctuates back 


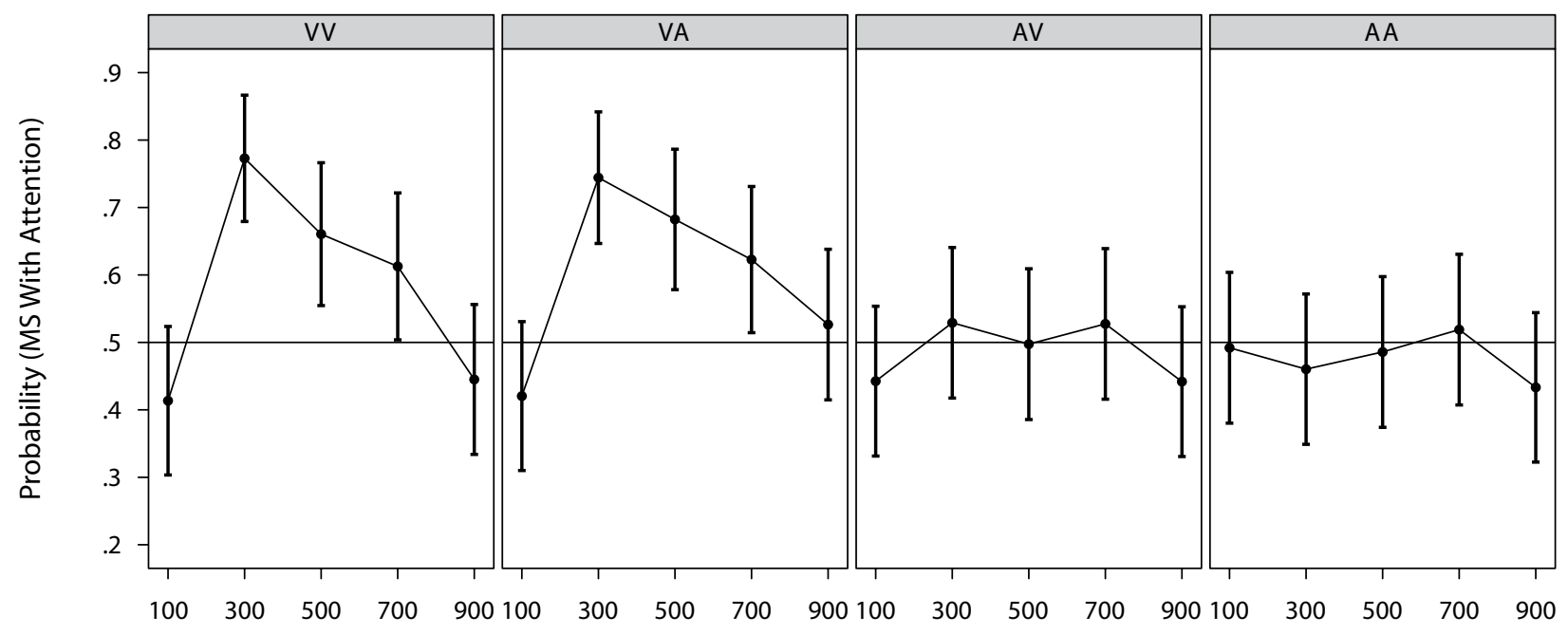

Microsaccade (MS) Time of Occurrence (msec)

Figure 5. Probability that microsaccade follows attention [i.e., $x=p($ MTC \& valid cues) -0.16$) / .48$ ] at centers of six 200-msec bins of microsaccade onset times in cue-target interval $(0-200,201-400$, etc.). $\mathrm{VV}=$ visual cue, visual target; $\mathrm{VA}=$ visual cue, auditory target; $\mathrm{AV}=$ auditory cue, visual target; $\mathrm{AA}=$ auditory cue, auditory target. Data are from four experiments reported in Rolfs et al. (2005). Graph produced with ggplot (R Development Core Team, 2009; Wickham, 2009).

to fixation or even in the opposite direction. This possibly reflects that attention goes back to fixation (Laubrock et al., 2005) or in the opposite direction (Rolfs, Engbert, \& Kliegl, 2004; see also Galfano et al., 2004, for uninformative cues). Alternatively, it could be indicative of an attempt to prevent premature responding.

Time window. The SA-MD link is strongest for microsaccades in the time window of highest cue-directed rates. The suppression-overshoot pattern has been found in virtually all studies of microsaccades in attentional cuing with human subjects (but not with monkeys, where the overshoot is missing; see, e.g., Cui et al., 2009). The peak itself appears to be sensitive to task demands: It shifts toward longer times when the task demands increase. Here we reported analyses from a very easy task with an early peak around $300 \mathrm{msec}$ and from a fairly difficult task with a late peak around $400 \mathrm{msec}$. The SA-MD link went with the peak.

Saccadic response stronger than manual response. We found the strongest SA-MD link for a saccadic response in an easy task with central informative cues. This link was stronger than for a manual response with the same experimental demand. We speculate that preparing for a saccadic response globally enhances the visual responsiveness in the superior colliculus, effectively driving the response system closer to threshold. Within the appropriate time window, the SA-MD link was of intermediate strength for manual responses; it was far from eliminated. Interestingly, for the visual-cue conditions of the cross-modal study, the link was of about the same magnitude despite the large differences in task difficulty.

Visual cues. The SA-MD link was found for visual cues but not for auditory cues. Interestingly, the modality of the target did not matter in the crossmodal study (see Figure 5). Although we would certainly not want to rule out that other auditory cues may trigger visual spatial attention, we consider the clear pattern of results reported here as a validation of the assumption that we are primarily looking at visual spatial attention.

We demonstrated that under specific experimental conditions and using specific selection criteria, microsaccades follow spatial attention in the CTI to a remarkably high degree. Why are some microsaccades affected by attention, whereas others are not? Although Bridgeman and Palca (1980) assumed in their pioneering work that "the function of microsaccades is unknown" (p. 817), later results indicate that microsaccades are the expression of ongoing physiological processes whose main functions might be maintenance of fixation (Engbert \& Kliegl, 2004) and prevention of perceptual fading (Engbert \& Mergenthaler, 2006; Martinez-Conde et al., 2006). We think that attention and other higher level cognitive processes (maybe due to their influence on collicular activity) are modulated upon the physiological signal expressed by these ongoing processes. Our results indicate that initial, cue-driven shifts of visual attention are likely to be expressed in microsaccade direction, whereas later processes of maintaining attention at the target are not expressed, at least not in the present task.

We need to qualify our results with respect to the horizontal cue-target arrangement. Given that microsaccades detected with our equipment and algorithm are predominantly horizontally oriented (Engbert, 2006, Figure 5), we think that potential biases of microsaccade orientation by vertical attention shifts will be much harder to detect.

Finally, a correlation of microsaccades with visuospatial attention within cuing paradigms does not necessarily mean that cues cause attention shifts, which in turn cause microsaccades. Alternatively, cues could independently cause attention shifts and microsaccades. Thus, it remains 
to be seen whether the attention-microsaccade relationship also holds for other forms of attention - for example, endogenous, top-down attention shifts in the absence of cues. These are inherently more variable, both temporally and interindividually; thus, their correlates will be harder to observe. Further research is needed to establish such effects. Some hints in the literature, such as results from ambiguous apparent motion perception, where reports of changes in the perceptual interpretation of ambiguous stimuli temporally follow microsaccades (Laubrock et al., 2008), or results from a visual oddball task, where microsaccade rate is modulated by task relevance and the proportion of targets (Valsecchi, Dimigen, Kliegl, Sommer, \& Turatto, 2009), suggest that such a relationship can in principle be expected.

One novel aspect of our data is that microsaccade amplitudes are modulated by whether or not they are related to visual attention. One might speculate that some microsaccades result from the inhibition of saccades. In summary, our results indicate that if the attentional signal is strong enough and not smeared out over time, then chances are that it will be expressed in microsaccade direction. Some, but not all microsaccades provide a window on visuospatial attention.

\section{AUTHOR NOTE}

We thank Todd Horowitz, Jeremy Wolfe, Bruce Bridgeman, and an anonymous reviewer for helpful comments on an earlier version of this article. This research was supported by Deutsche Forschungsgemeinschaft (Grants KL-955/3 and KL/955-6). Data and R scripts are available upon request. Correspondence related to this article may be sent to J. Laubrock or R. Kliegl, Department of Psychology, University of Potsdam, Karl-Liebknecht-Str. 24-25, 14476 Potsdam, Germany (e-mail: laubrock@uni-potsdam.de or kliegl@uni-potsdam.de).

\section{REFERENCES}

Bates, D., \& MaEChLER, M. (2009). lme4: Linear mixed-effects models using S4 classes (R package version 0.999375-31) [Software]. Available at http://CRAN.R-project.org/package $=1 \mathrm{me} 4$

Brainard, D. H. (1997). The Psychophysics Toolbox. Spatial Vision, 10, 433-436. doi:10.1163/156856897X00357

Bridgeman, B., \& PALCA, J. (1980). The role of microsaccades in high acuity observational tasks. Vision Research, 20, 813-817. doi:10.1016/ 0042-6989(80)90013-9

Corneil, B. D., Munoz, D. P., Chapman, B. B., Admans, T., \& CushING, S. L. (2008). Neuromuscular consequences of reflexive covert orienting. Nature Neuroscience, 11, 13-15. doi:10.1038/nn2023

Cornelissen, F. W., Peters, E. M., \& Palmer, J. (2002). The Eyelink Toolbox: Eye tracking with MATLAB and the Psychophysics Toolbox. Behavior Research Methods, Instruments, \& Computers, 34, 613-617.

Cui, J., Wilke, M., Logothetis, N. K., Leopold, D. A., \& Liang, H. (2009). Visibility states modulate microsaccade rate and direction. Vision Research, 49, 228-236. doi:10.1016/j.visres.2008.10.015

Deubel, H., \& SchneIDER, W. X. (1996). Saccade target selection and object recognition: Evidence for a common attentional mechanism. Vision Research, 36, 1827-1837. doi:10.1016/0042-6989(95)00294-4

ENGBERT, R. (2006). Microsaccades: A microcosm for research on oculomotor control, attention, and visual perception. Progress in Brain Research, 154, 177-192. doi:10.1016/S0079-6123(06)54009-9

Engbert, R., \& KLIEgL, R. (2003). Microsaccades uncover the orientation of covert attention. Vision Research, 43, 1035-1045. doi:10.1016/ S0042-6989(03)00084-1

Engbert, R., \& Kliegl, R. (2004). Microsaccades keep the eyes' balance during fixation. Psychological Science, 15, 431-436. doi:10.1111/j.0956-7976.2004.00697.x

Engbert, R., \& Mergenthaler, K. (2006). Microsaccades are trig- gered by low retinal image slip. Proceedings of the National Academy of Sciences, 103, 7192-7197. doi:10.1073/pnas.0509557103

FindLAY, J. M., \& GILCHRIST, I. D. (2003). Active vision: The psychology of looking and seeing. Oxford: Oxford University Press.

Galfano, G., Betta, E., \& Turatto, M. (2004). Inhibition of return in microsaccades. Experimental Brain Research, 159, 400-404. doi:10.1007/s00221-004-2111-y

Gallistel, C. R. (1990). The organization of learning. Cambridge, MA: MIT Press.

Gowen, E., Abadi, R. V., Poliakoff, E., Hansen, P. C., \& Miall, R. C. (2007). Modulation of saccadic intrusions by exogenous and endogenous attention. Brain Research, 1141, 154-167. doi:10.1016/ j.brainres.2007.01.047

HAFED, Z. M., \& Clark, J. J. (2002). Microsaccades as an overt measure of covert attention shifts. Vision Research, 42, 2533-2545. doi:10.1016/S0042-6989(02)00263-8

Hafed, Z. M., Goffart, L., \& Krauzlis, R. J. (2009). A neural mechanism for microsaccade generation in the primate superior colliculus. Science, 323, 940-943.

Hoffman, J. E., \& Subramaniam, B. (1995). The role of visual attention in saccadic eye movements. Perception \& Psychophysics, 57, 787-795.

Horowitz, T. S., Fencsik, D. E., Fine, E. M., Yurgenson, S., \& WoLFE, J. M. (2007). Microsaccades and attention: Does a weak correlation make an index? Reply to Laubrock, Engbert, Rolfs, \& Kliegl (2007). Psychological Science, 18, 367-368. doi:10.1111/j.1467 $-9280.2007 .01905 . \mathrm{x}$

Horowitz, T. S., Fine, E. M., Fencsik, D. E., Yurgenson, S., \& Wolfe, J. M. (2007). Fixational eye movements are not an index of covert attention. Psychological Science, 18, 356-363. doi:10.1111/j.1467 -9280.2007.01903.x

HsIEH, P.-J., \& TsE, P. U. (2009). Microsaccade rate varies with subjective visibility during motion-induced blindness. PLoS One, 4, e5163. doi:10.1371/journal.pone. 0005163

Jonides, J. (1980). Towards a model of the mind's eye's movement. $\mathrm{Ca}$ nadian Journal of Psychology, 34, 103-112.

Kliegl, R., Rolfs, M., Laubrock, J., \& Engbert, R. (2009). Microsaccadic modulation of response times in spatial attention tasks. Psychological Research, 73, 136-146. doi:10.1007/s00426-008-0202-2

Kohama, T., \& Usui, S. (2002). Attentional effects on microsaccadic eye movements. Current Psychology of Cognition, 21, 377-395.

Kowler, E., Anderson, E., Dosher, B., \& Blaser, E. (1995). The role of attention in the programming of saccades. Vision Research, 35, 1897-1916. doi:10.1016/0042-6989(94)00279-U

Laubrock, J., Engbert, R., \& Kliegl, R. (2005). Microsaccade dynamics during covert attention. Vision Research, 45, 721-730. doi:10.1016/j.visres.2004.09.029

Laubrock, J., EnGbert, R., \& Kliegl, R. (2008). Fixational eye movements predict the perceived direction of ambiguous apparent motion. Journal of Vision, 8(14, Art. 13), 1-17. doi:10.1167/8.14.13

Laubrock, J., Engbert, R., Rolfs, M., \& Kliegl, R. (2007). Microsaccades are an index of covert attention. Commentary on Horowitz, Fine, Fencsik, Yurgenson, and Wolfe (2007). Psychological Science, 18, 364-366. doi:10.1111/j.1467-9280.2007.01904.x

Martinez-Conde, S., Macknik, S. L., Troncoso, X. G., \& Dyar, T. A. (2006). Microsaccades counteract visual fading during fixation. Neuron, 49, 297-305. doi:10.1016/j.neuron.2005.11.033

Martinez-Conde, S., Macknik, S. L., Troncoso, X. G., \& Hubel, D. H. (2009). Microsaccades: A neurophysiological analysis. Trends in Neurosciences, 32, 463-475. doi:10.1016/j.tins.2009.05.006

Mergenthaler, K., \& Engbert, R. (2007). Modeling the control of fixational eye movements with neurophysiological delays. Physical Review Letters, 98, 138104: 1-4. doi:10.1103/PhysRevLett.98.138104

Pelli, D. G. (1997). The VideoToolbox software for visual psychophysics: Transforming numbers into movies. Spatial Vision, 10, 437-442.

PosNer, M. I. (1980). Orienting of attention: The VIIth Sir Frederic Bartlett lecture. Quarterly Journal of Experimental Psychology, 32A, 3-25. doi:10.1080/00335558008248231

Posner, M. I., SNyder, C. R., \& Davidson, B. J. (1980). Attention and the detection of signals. Journal of Experimental Psychology: General, 109, 160-174.

R Development Core Team (2009). R: A language and environment for statistical computing [Software]. Vienna: R Foundation for Statistical Computing. Available at www.R-project.org. 
Rolfs, M. (2009). Microsaccades: Small steps on a long way. Vision Research, 49, 2415-2441. doi:10.1016/j.visres.2009.08.010

Rolfs, M., Engbert, R., \& Kliegl, R. (2004). Microsaccade orientation supports attentional enhancement opposite a peripheral cue. Psychological Science, 15, 705-707. doi:10.1111/j.09567976.2004.00744.x

Rolfs, M., Engbert, R., \& Kliegl, R. (2005). Crossmodal coupling of oculomotor control and spatial attention in vision and audition. Experimental Brain Research, 166, 427-439. doi:10.1007/s00221 $-005-2382-y$

Rolfs, M., Kliegl, R., \& Engbert, R. (2008). Toward a model of microsaccade generation: The case of microsaccadic inhibition. Journal of Vision, 8(11, Art. 5), 1-23. doi:10.1167/8.11.5

STARZYNSKI, C., \& ENGBERT, R. (2009). Noise-enhanced target discrimination under the influence of fixational eye movements and external noise. Chaos, 19, 015112. doi:10.1063/1.3098950

Troncoso, X. G., Macknik, S. L., \& Martinez-Conde, S. (2008). Microsaccades counteract perceptual filling-in. Journal of Vision, 8(14, Art. 15), 1-9. doi:10.1167/8.14.15

Troncoso, X. G., Macknik, S. L., Otero-Millan, J., \& MartinezConde, S. (2008). Microsaccades drive illusory motion in the enigma illusion. Proceedings of the National Academy of Sciences, 105, 16033-16038. doi:10.1073/pnas.0709389105

Turatto, M., Valsecchi, M., Tamè, L., \& Betta, E. (2007). Microsaccades distinguish between global and local visual processing. NeuroReport, 18, 1015-1018. doi:10.1097/WNR.0b013e32815b615b

Valsecchi, M., Dimigen, O., Kliegl, R., Sommer, W., \& Turatto, M. (2009). Microsaccadic inhibition and P300 enhancement in a visual oddball task. Psychophysiology, 46, 635-644. doi:10.1111/j.1469 -8986.2009.00791.x

VAN DAM, L. C. J., \& VAN EE, R. (2006). Retinal image shifts, but not eye movements per se, cause alternations in awareness during binocular rivalry. Journal of Vision, 6, 1172-1179. doi:10.1167/6.11.3

van Gisbergen, J. A. M., Robinson, D. A., \& Gielen, S. (1981). A quantitative analysis of generation of saccadic eye movements by burst neurons. Journal of Neurophysiology, 45, 417-442.

WiскНАм, H. (2009). ggplot2: Elegant graphics for data analysis. New York: Springer.

\section{NOTE}

1. Why should attention not always follow the cue - that is, $w<1.00$ ? The case of $w=1.00$ indicates subjects who perform according to Bayesian optimality given knowledge of unchanging cue validity. There are both empirical (Jonides, 1980) and theoretical (Gallistel, 1990) reasons for why subjects in a potentially unstable environment are often likely to allocate their attention somewhat more in accordance with probability matching. Furthermore, due to fluctuations of vigilance, it is likely that the limiting case of $w=1$ is hardly ever reached in reality. Although we cannot directly observe $w$, the usually robust cue validity effects in RT assure us that we can probably safely assume that $.80<w<1.00$; that is, the true probability that subjects shift their attention with the cue lies between probability matching $(w=.80)$ and Bayesian optimality $(w=1)$.

(Manuscript received August 14, 2008; revision accepted for publication November 4, 2009.) 